\title{
Optical Properties of Thin Films on Transparent Surfaces by Ellipsometry; Internal Reflection for Film Covered Surfaces Near the Critical Angle
}

\author{
Elio Passaglia and Robert R. Stromberg
}

(August 5, 1964)

\begin{abstract}
The application of ellipsometry to the determination of the optical properties of thin films on transparent substrates by the use of internal reflection and angles of incidence near the critical angle for total reflection is described and illustrated. Four cases are considered: 1. the angle of incidence, $\theta_{i}$, is less than either the critical angle for total reflection between the substrate and the film, $\theta_{c}{ }^{1,2}$, and the critical angle between the substrate and the surrounding medium, $\theta_{c}^{1,3} ; 2 . \theta_{c}^{1,3}>\theta_{i}>\theta_{c}^{1,2} ; 3 . \theta_{c}^{1,3}<\theta_{i}<\theta_{c}^{1,2}$; and $4 . \theta_{c}{ }^{1,3}<\theta_{i}>\theta_{c}{ }^{1,2}$. For case 1 , at certain critical values of film thickness $d$ and refractive index $n_{2}$, the reflected light may be polarized with its electric vector either entirely in or normal to the plane of incidence. Near these conditions the sensitivity of ellipsometric measurements is extremely high, but the intensity of the reflected light is very low. Except under these conditions the intensity is adequate for experimental measurements, as it is also in case 2 . For case 1 it is always possible to determine $n_{2}$ and $d$ by a single measurement; for case 2 this is possible only tor thin films. For cases 3 and 4, and case 2 for thick films, only one of these may be determined. Under these conditions, however, the reflectivity is 100 percent.
\end{abstract}

\section{Introduction}

The determination of the optical constants of surfaces and the thickness and refractive index of thin films by measurement of the changes in the state of polarization of polarized light upon reflection is based on the well-known equations of Drude [1]. ${ }^{1} \quad$ The technique for carrying out these measurements is frequently referred to as ellipsometry and has been described in detail elsewhere [e.g., 2, 3, 4].

Most work with ellipsometry has been concerned with studies on metallic surfaces [2,3], either because of their intrinsic interest or because they form highly reflecting substrates for the study of thin films. However, it is sometimes desirable to measure the properties of thin films on transparent surfaces, in which case the low intensity of the reflected light makes accurate analysis of its state of polarization difficult.

There are two possible systems which would, in general, increase the intensity of the reflected beam over that obtained by direct external reflection from a transparent surface. In one, a thin layer of the transparent material which is to be used as a substrate for the film is placed in intimate contact with a metal, which is used as the reflecting substrate. The transparent material may be placed as a film on a metallic plate, for example by evaporating a film of silica on a metallic substrate in a vacuum evaporator.

1 Figures in brackets indicate the literature reference at the end of this paper.
It is necessary, of course, to determine the optical properties of the metal prior to deposition of the film, and these properties must not change as a result of this deposition. The metal may also be evaporated onto the transparent material. In this case it should be possible to determine the optical properties of the bare metallic surface directly, and, from the other side, the properties of the transparent plate. For this technique the faces of the plate must be sufficiently flat and parallel. This system of a transparent film backed in some manner by a metallic reflecting surface does not differ from the usual system of a metallic reflector with multiple films [5], and will not be discussed further.

A second possible system is one in which it is arranged to have internal reflection at the boundary of the transparent material in question and to use angles near or above the critical angle for total internal reflection. This can be accomplished by constructing a prism of the substrate material or, if other considerations permit, by placing the material in a liquid of higher refractive index. At angles of incidence greater than the critical angle, all the light is reflected and intensity problems are obviated. Although there is total reflection at angles of incidence greater than the critical angle, the beam does penetrate the medium of lower refractive index; this effect has been used to study the infrared spectra of opaque materials $[6,7]$. In ellipsometric applications, total reflection permits the use of multiple reflections to increase sensitivity [8], but does lead to the serious limitation that the refractive index and thickness of a thin film may not be determined simultaneously. 


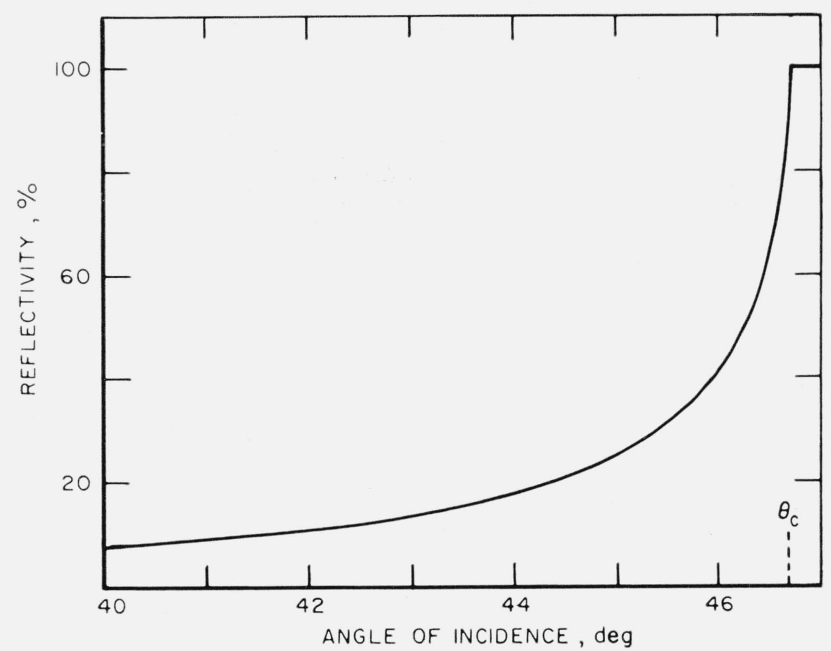

FIGURE 1. Reflectivity as a function of angle of incidence for internal reflection in the neighborhood of the critical angle.

The calculations were made using the refractive indexes given in table 1.

At angles between the polarizing angle and the critical angle the intensity of light reflected rises very sharply as the critical angle is approached, and this region may be used quite effectively for ellipsometric studies. For example, the intensity of reflected light as a function of angle of incidence is shown in figure 1 (critical angle $=46.68 \mathrm{deg}$ ). For an angle of incidence within $0.2 \mathrm{deg}$ of the critical angle, the reflectivity is 60 percent. Although the reflectivity will vary greatly with the thickness and refractive index of any film on the surface, it will be shown that the reflectivity remains quite high for relatively thin films and hence this is a useful region for carrying out ellipsometric measurements on thin films on transparent substrates.

This paper will illustrate the application of ellipsometry to this type of system by presenting detailed calculations of the changes in the state of polarization of light reflected from a transparent surface covered by a transparent film for certain specific values of angles of incidence and refractive indexes. The calculations were carried out by means of the program described by McCrackin and Colson [9] using the IBM 7094 computer.

\section{Reflection at an Interface}

As is well known, $\rho$, the ratio of the reflection coefficient for light polarized with the electric vector in the plane of incidence, $r^{p}$, to the reflection coefficient for light polarized with the electric vector normal to the plane of incidence, $r^{s}$, may be represented by the equation

$$
\rho=\frac{r^{p}}{r^{s}}=\tan \psi e^{i \Delta}
$$

where $\tan \psi$ is the ratio of the magnitude of the two coefficients and $\Delta$ is the difference in their phases.
For a bare surface $\rho$ is simply the ratio of the Fresnel coefficients. For a semi-infinite medium (which we call the "medium") of refractive $n_{3}$ separated by a film of refractive index $n_{2}$ from another semi-infinite medium of refractive index $n_{1}$ (which we call the "substrate"), the ratio of reflection coefficients for light incident at the $n_{1}-n_{2}$ interface from the $n_{1}$ side is given by the exact Drude equation

$$
\tan \psi e^{i \Delta}=\frac{\frac{r_{12}^{p}+r_{23}^{p} \exp D}{1+r_{12}^{p} r_{23}^{p} \exp D}}{\frac{r_{12}^{s}+r_{23}^{s} \exp D}{1+r_{12}^{s} r_{s 3}^{2} \exp D}}
$$

where $r_{12}$ and $r_{23}$ are the Fresnel coefficients at the substrate-film and film-medium interface respectively. $\quad D$ is given by

$$
D=-4 \pi i n_{2} \cos \theta_{2} d / \lambda
$$

where $\theta_{2}$ is the angle of incidence at the film-medium interface, $\lambda$ is the wavelength of the light in vacuum, and $i=\sqrt{-1}$. It is clear that if all the parameters in this equation are known but two, say $n_{2}$ and $d$ (provided $n_{2}$ is real), then a measurement of $\Delta$ and $\psi$ permits the determination of these two, and the methods for doing this have been described elsewhere $[2,3]$.

If total reflection occurs, however, $r^{p}$ and $r^{s}$ both have a magnitude of unity, so that $\psi$ is always equal to $\pi / 4$. This gives only one experimental parameter $(\Delta)$ and permits the calculation of only one of the quantities $n_{2}$ or $d$; the other must be determined by other means. This is the most serious limitation imposed by total internal reflection.

\section{Parameters of Systems Discussed}

We consider only the case in which $n_{1}$ is greater than both $n_{2}$ and $n_{3}$ and all indexes are real. Since light is considered incident at the $n_{1}-n_{2}$ interface from the higher refractive index side, internal reflection occurs. We call the angle of incidence $\theta_{i}$, and for such a system two critical angles must be considered. These are $\theta_{c}^{1,2}$, the critical angle for total reflection between the substrate and the film, and $\theta_{c}^{1,3}$, the critical angle for total reflection between the substrate and the surrounding medium. Four cases can then be considered: one in which the angle of incidence, $\theta_{i}$, is less than either $\theta_{c}^{1,2}$ or $\theta_{c}^{1,3}$, two cases in which $\theta_{i}$ is greater than one of these but less than the other, and the case in which $\theta_{i}$ exceeds both these critical angles. Note that the critical angle for reflection at the film-medium interface, $\theta_{c}^{2,3}$, need not be specifically considered, for when $\theta_{i}=\theta_{c}^{1,3}$, the angle of incidence at the film-medium interface, $\theta_{i}^{2,3}$, is equal to $\theta_{c}^{2,3}$, and if $\theta_{i}$ is greater or less than $\theta_{c}^{1,3}, \theta_{i}^{2,3}$ will automatically be greater or less than $\theta_{c}^{2,3}$.

The parameters of the system chosen for calculation are given in table 1 . The values for $n_{1}$ and $n_{3}$ arose out of an experimental study reported elsewhere [10]. The index $n_{1}$ pertains to a leaded glass 
prism immersed in a very dilute solution of an organic compound in water $(n=1.337)$. The adsorption of this compound onto the glass prism was studied by eilipsometry. Calculations have been carried out for the values of film refractive indexes $n_{2}$ shown in the table. The value of 1.20 , while not easy to achieve experimentally, was chosen merely to illustrate the behavior when $n_{2}<n_{3}$.

In all the cases shown we shall be interested in computing the experimentally obtainable quantities $\psi$ and $\Delta$ as a function of film thickness. We shall also be interested in the amount of light reflected, and will consider each case in turn.

TABLE 1. Parameters of system chosen for calculation $\lambda=5461 \AA$ $\begin{array}{ll}n_{1}=1.8376 & \theta_{c}{ }^{1,3}=46.68 \mathrm{deg} \\ n_{3}=1.3370 & \theta_{p^{1,3}}=36.05 \mathrm{deg} *\end{array}$

\begin{tabular}{|c|c|c|c|c|c|}
\hline Case No. & & $\theta_{i}$, deg & $n_{2}$ & $\theta_{c}^{1,2}, \operatorname{deg}$ & $\theta_{p}^{1,2}, \operatorname{deg}$ \\
\hline 1 & $\begin{aligned} \theta_{i} & <\theta_{c}{ }_{c}^{1,2} \\
& <\theta_{c}^{1,3}\end{aligned}$ & $\begin{array}{l}46.00 \\
46 . € 0\end{array}$ & $\begin{array}{l}1.60-1.36 \\
1.38-1.36\end{array}$ & $\begin{array}{l}60.7-47.8 \\
48.6-47.8\end{array}$ & $\begin{array}{l}41.1-36.5 \\
36.9-36.5\end{array}$ \\
\hline 2 & $\begin{aligned} \theta_{i} & \geqslant \theta_{c}{ }^{1,2} \\
& <\theta_{c}^{1,3}\end{aligned}$ & 46.00 & $\begin{array}{l}\text { 1. } 200 \text {, } \\
1.300\end{array}$ & $\begin{array}{l}40.8 \\
45.0\end{array}$ & 33.3 \\
\hline 3 & $\begin{array}{r}\theta_{i}<\theta_{c} c^{1,2} \\
>\theta_{c}{ }^{1,3}\end{array}$ & 46.70 & $\begin{array}{l}2.00 \\
1.60 \\
1.40 \\
1.35\end{array}$ & $\begin{array}{l}60.7 \\
49.6 \\
47.2\end{array}$ & $\begin{array}{l}41.1 \\
37.3 \\
36.3\end{array}$ \\
\hline 4 & $\begin{aligned} \theta_{i} & >\theta_{c}{ }^{1,2} \\
& >\theta_{c}{ }^{1,3}\end{aligned}$ & $\begin{array}{l}46.70 \\
50.00 \\
50.00\end{array}$ & $\begin{array}{l}1.20 \\
1.40 \\
1.20\end{array}$ & $\begin{array}{l}40.8 \\
49.6 \\
40.8\end{array}$ & $\begin{array}{l}33.2 \\
37.3 \\
33.3\end{array}$ \\
\hline
\end{tabular}

${ }^{*} \theta_{p}$ denotes the polarizing (Brewester's) angle.

\section{Case $1, \theta_{c}^{1,3}>\theta_{i}<\theta_{c}^{1,2}$}

In this case, when the angle of incidence is less than either of the two critical angles, some interesting polarization effects occur. This is due to the fact that for certain critical values of thickness and refractive index of the film $r^{s}$ or $r^{p}$ may go to zero. When this occurs the reflected light is polarized with its electric vector in the plane of incidence $\left(r^{s}=0\right)$ or normal to the plane of incidence $\left(r^{p}=0\right)$. The value of $\tan \psi$ may therefore be anything from zero $\left(r^{p}=0\right)$ to infinity $\left(r^{s}=0\right)$. The vanishing of $r^{p}$ for dielectric films on dielectric substrates is well known $[11,12]$, and the vanishing of $r^{p}$ and $r^{s}$ for dielectric films on metal surfaces has been discussed by Hartman [13] and Mattuck [14]. The conditions for the vanishing of $r^{s}$ for dielectric films on dielectric substrates have not, to our knowledge, been previously studied. In the appendix there are derived the conditions for the vanishing of $r^{s}$ and $r^{p}$. It is shown there that for each case the optical thickness of the film must be such that the phase retardation in the film is an odd multiple of $\pi$, i.e.,

$$
\frac{2 n_{2} d \cos \theta_{2}}{\lambda}=\frac{k}{2}
$$

where $k$ is an odd integer. We shall call the thickness necessary to fulfill this condition the "quarter wave thickness." For this thickness $\Delta$ is zero or $\pi$, and if the incident light is linearly polarized, the reflected is also.
In addition to this condition, there is a condition on $n_{2}$ for the vanishing of $r^{s}$ or $r^{p}$. We shall discuss the former case first.

For the vanishing of $r^{s}$, it is shown in the appendix that $n_{2}$ must assume a critical value, $n_{2 s}$, given by the equation

$$
n_{2 s}^{2}=n_{1} n_{3} \cos \theta_{1} \cos \theta_{3}+n_{1}^{2} \sin ^{2} \theta_{1}
$$

where the symbols have the obvious meanings. For an angle of incidence of $46.00 \mathrm{deg}$ and the other parameters as in table $1, n_{2 s}=1.41542$, and the corresponding thickness from eq (3) is $2700 \AA$. For these values of the film refractive index and thickness the reflected light will be linearly polarized, but, unlike the condition at Brewster's angle, the electric vector is in the plane of incidence, not normal to it. Thus $\tan \psi$ becomes infinite. We shall now discuss the behavior of $\rho$, the ratio of reflection coefficients (see eq (1)) with film thickness for two values of refractive index near the critical index $n_{2 s}$.

A polar plot of $\rho$ is given in figure 2 for $n_{2}$ values of 1.41485 and 1.41600 , i.e., slightly lower and slightly higher than $n_{2 s}$. The numbers on the curves correspond to film thicknesses. While the curve for $n_{2}=1.41600$ lies entirely to right of the origin, the curve for $n_{2}=1.41485$ goes around the origin. (The very large values of $\tan \psi$ for these cases make this somewhat hard to see, and therefore, an enlargement of the region around the origin is shown separately. The value of $\psi$ for these curves at zero thickness is, of course, a constant determined by $\theta_{i}, n_{1}$, and $n_{3}$.) That is to say, for $n_{2}$ values above the critical value, as film thickness increases $\Delta$ oscillates between two extreme values, $\Delta_{\max }$ and $\Delta_{\min }$, symmetrically disposed with respect to the value of $\Delta$ at zero film thickness. For values of $n_{2}$ less than $n_{2 n}, \Delta$ assumes all values from zero to $2 \pi$.

At the quarter wave thickness the waves reflected from the 2, 3 interface and transmitted into medium 1 are $\pi$ radians out of phase with both the incident wave and the wave reflected from the 1, 2 interface. For $n_{2}>n_{2 s}$ the amplitude of the sum of the $s$ waves reflected from the 2, 3 interface and transmitted into medium 1 is less than the amplitude of the $s$ wave reflected at the 1,2 interface, and hence the total reflected $s$ wave is in phase with the incident wave. For $n_{2}<n_{2 s}$, the amplitude of the sum of the reflected and retransmitted $s$ waves is greater than the amplitude of the $s$ wave reflected at the 1,2 interface, and the total reflected $s$ wave is $\pi$ radians out of phase with the incident wave. Thus $\Delta$ at the quarter wave thickness changes from 0 to $\pi$ as $n_{2}$ goes from above $n_{2 s}$ to below.

There is an extremely great dependence of $\Delta$ upon film thickness for values of $n_{2}$ near the critical refractive index $n_{2 s}$ and for film thicknesses near the quarter-wave thickness, i.e., when $\Delta=0$ for $n_{2}>n_{2 s}$ or $\pi$ for $n_{2}<n_{2 s}$. Thus, for example, from the curve for $n_{2}=1.41600$, a change of thickness from 2688 to $2690 \AA$ produces a change in $\Delta$ of $22 \mathrm{deg}$. This is a change of 11 deg per angstrom. Clearly, ellipsometric measurements under these conditions are exceedingly sensitive to thickness. 


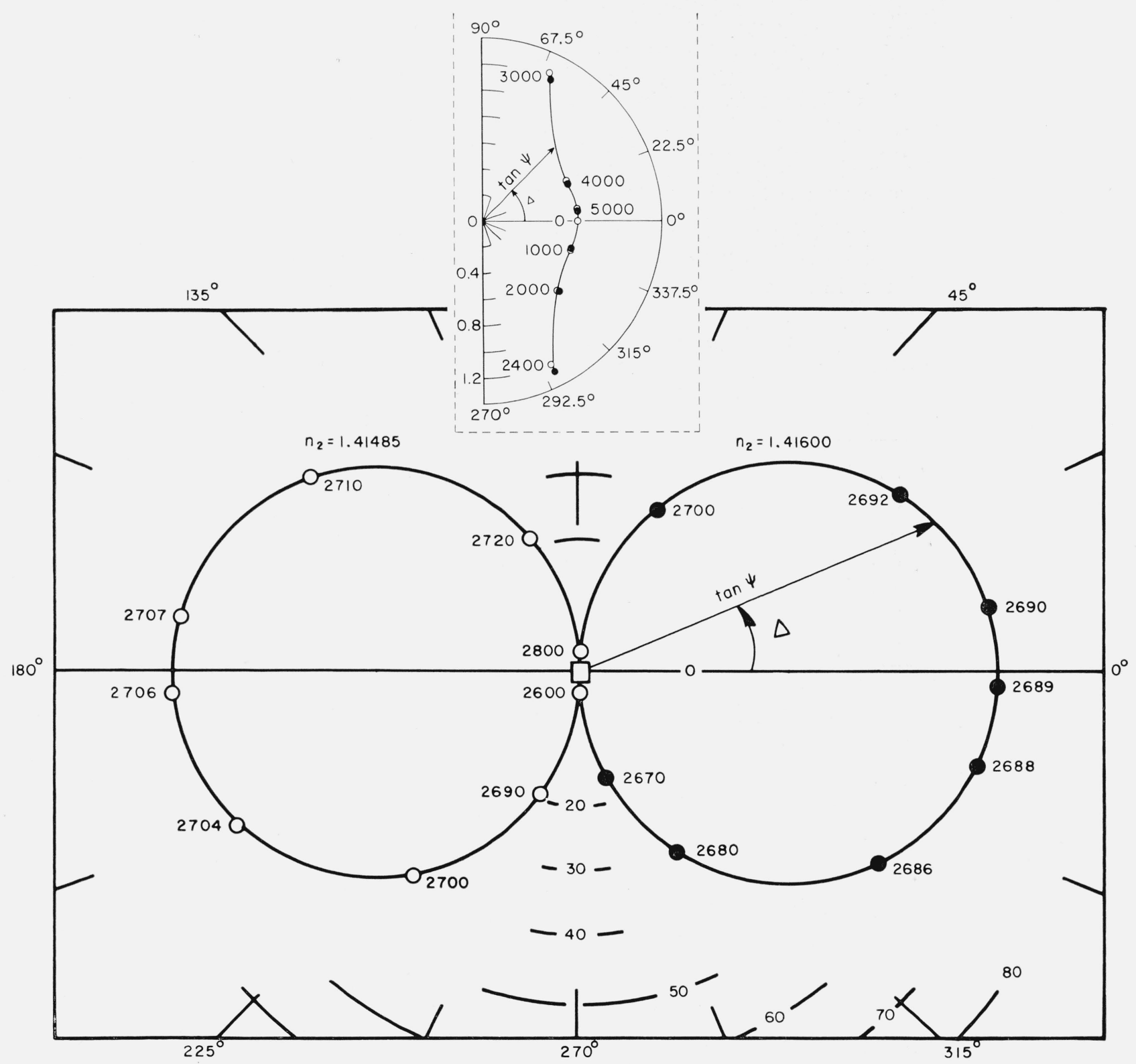

Figure 2. Case 1. A polar plot of $\rho$ for $\mathrm{n}_{2}$ values slightly lower and slightly higher than $\mathrm{n}_{2 \mathrm{~s}}($ see text). The insert is an enlargement of the rectangular area at the center. The numbers on the curves correspond to film thickness. Other parameters as in table 1.

The dependence of $\psi$ on thickness is less easy to see from the figure. The calculations indicate that for a film refractive index of 1.41540 , i.e., very close to $n_{2 s}$, a change in thickness from 2694 to $2698 \AA$ changes $\tan \psi$ from 80 to 1250 . This extreme dependence of $\tan \psi$ on thickness indicates that the production of accurately linearly polarized light by this method would be a difficult experimental feat. Moreover, the intensity of light reflected under these polarizing conditions is very low, but we sball defer discussion of this until later in this paper.
We shall now discuss the behavior of $\rho$ when $r^{p}$ vanishes. For this to occur, eq (3) must be obeyed, and in addition there is a condition on $n_{2}$. As shown in the appendix, the values of $n_{2}$ necessary to have the $r^{p}$ vanish, which we call $n_{2 p}$, are given by

$$
n_{2 p}^{2}=\frac{1}{2} \frac{n_{1} n_{3}}{\cos \theta_{1} \cos \theta_{3}}\left[1 \pm\left(1-\sin 2 \theta \sin 2 \theta_{3}\right)\right]^{1 / 2}
$$

There are two values of $n_{2 p}$, corresponding to the positive and negative signs, and we call these 
$n_{2 p}^{+}$and $n_{2 p}^{-}$. For an angle of incidence of $46.00^{\circ}$ and $n_{1}$ and $n_{3}$ as shown in the table, $n_{2 p}^{+}$is 4.654 and out of the range of our consideration. However, $n_{2 p}^{-}$is 1.3786 , and the corresponding thickness from eq (3) is $3487 \AA$. For these values of thickness and refractive index the reflected light is polarized entirely with its electric vector normal to the plane of incidence, and $\tan \psi=0$.

Figure 3 shows the behavior of $\rho$ with film thickness for $n_{2}=n_{2 p}^{-}$and two other values near this value (1.36 and 1.3942). For values of $n_{2}$ less than $n_{2 p}^{-}$(but of course greater than $n_{3}$ ) the $\rho$ curve lies between the origin and the value of $\tan \psi$ for the bare surface (fig. 3, $n_{2}=1.36$ ). Again $\Delta$ oscillates between two values, $\Delta_{\max }$ and $\Delta_{\min }$, symmetrically disposed with respect to the value for the bare surface. For $n_{2}$ equal to $n_{2 p}^{-}$, the curve of $\rho$ passes through the origin $(\tan \psi=0)$ and $\Delta_{\max }$ and $\Delta_{\min }$ are $\pi / 2$ and $-\pi / 2$ respectively (fig. $3, n_{2}=1.3786$ ). For values of $n_{2}$ larger than $n_{2 p}^{-}$(but smaller than $n_{2 s}$ ) $\Delta$ takes on all values between 0 and $2 \pi$ (fig. $3, n_{2}=$ 1.3942).
For the quarter wave thickness and values of $n^{2}$ between $n_{2 p}^{-}$and $n_{2 s}$ the sum of the amplitudes of the waves reflected from the 2,3 interface and transmitted back into medium 1 is greater than the amplitude of the wave reflected from the 1,2 interface for $s$ waves, but less for $p$ waves. Thus the total reflected $s$ wave is $\pi$ radians out of phase with the incident wave. For angles of incidence greater than the polarizing angle the reflected $p$ wave however is in phase [2] and $\Delta$ is therefore $\pi$. For $n_{2}<n_{2 p}^{-}$, however, the sum of the amplitudes of the waves reflected from the 2,3 interface and transmitted back into medium 1 is greater than the amplitude of the wave reflected from the 1,2 interface for both the $s$ and $p$ components. Thus the reflected $s$ and $p$ waves are both $\pi$ radians out of phase with the incident wave and $\Delta$ is zero.

It is now easy to reconstruct the behavior of the reflection coefficient ratio for all values of $n_{2}$ between $n_{1}$ and $n_{3}$. For values of $n_{2}$ slightly less than $n_{1}$, such as the $n_{2}=1.60$ curve in figure 3 , the $\rho$ curve lies entirely to the right of the bare-surface point. In this

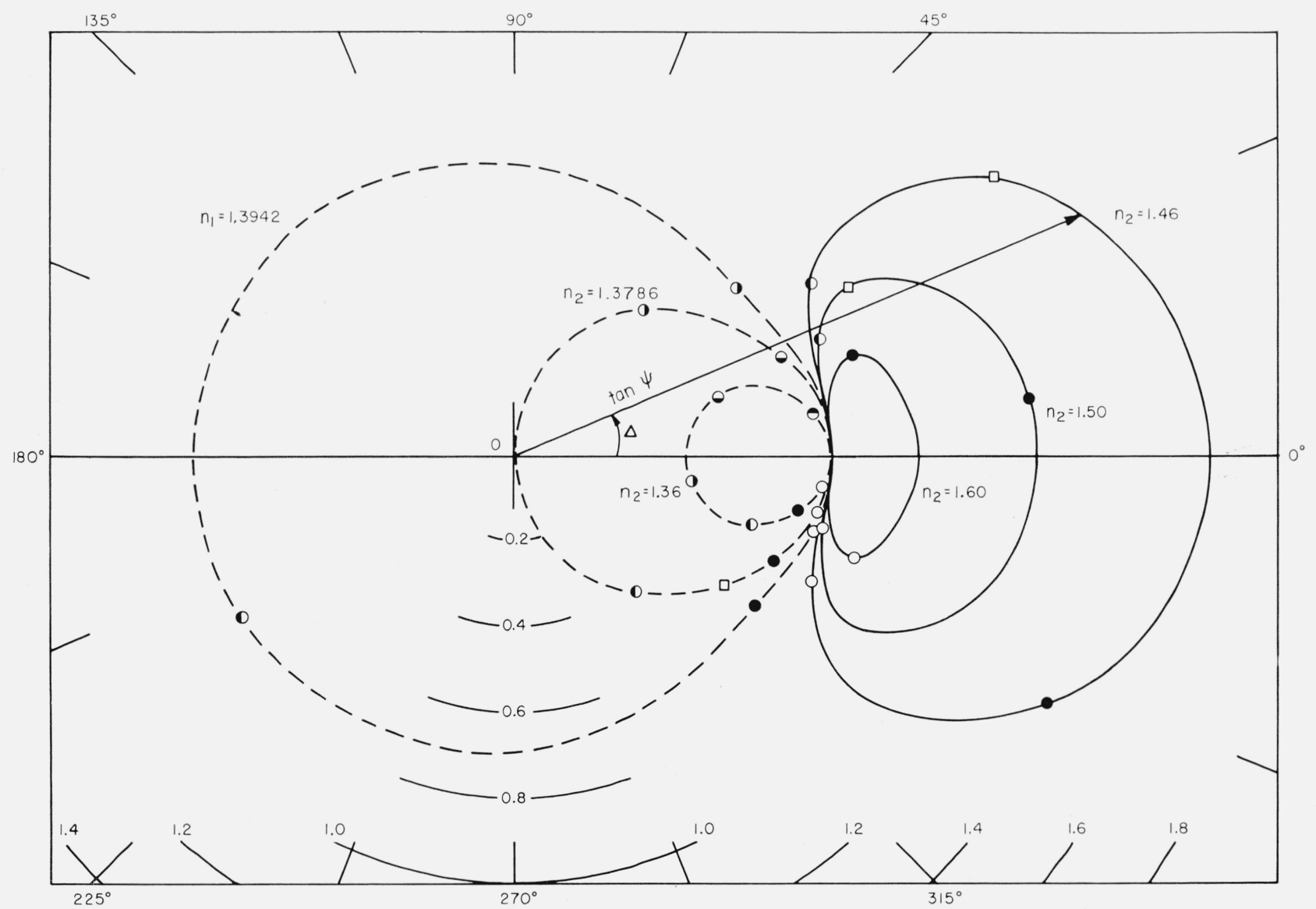

Figure 3. Case 1. Polar plot of $\rho$ for several values of $\mathrm{n}_{2}$ between $\mathrm{n}_{1}$ and $\mathrm{n}_{3}$.

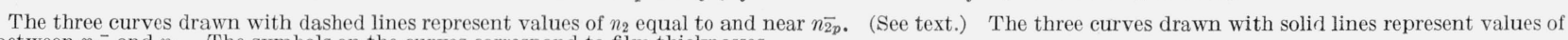
$n_{2}$ between $n_{2 p}$ and $n_{3}$. The symbols on the curves correspond to film thicknesses. 
region $\tan \psi$ oscillates between a minimum value for the bare surface and a maximum value at the quarter-wave thickness (at which $\Delta=0$ ) and $\Delta$ in turn oscillates between two values, $\Delta_{\max }$ and $\Delta_{\min }$. As the refractive index decreases, the maximum value of $\tan \psi$ increases, as do $\Delta_{\max }$ and $\Delta_{\min }$. (fig. $3, n_{2}=1.50$ and $n_{2}=1.46$ ), approaching infinity and $\pm \pi / 2$, respectively as $n_{2}$ approaches $n_{2 s}$ (fig. $2, n_{2}=1.416$ ). For values of $n_{2}$ less than $n_{2 s}$ but greater than $n_{2 p}^{-}, \Delta$ takes on all values between zero and $2 \pi$, but the maximum value of $\tan \psi$ (occurring when $\Delta=\pi$ ) decreases as $n_{2}$ becomes progressively lower (fig. 2, $n_{2}=1.41485$ and fig. $3, n_{2}=1.3942$ ). At some refractive index (equal to 1.3942 in the present case) between $n_{2 p}^{-}$and $n_{2 s}$ the value of $\tan \psi$ for $\Delta=\pi$ is equal to the value for the bare surface. As $n_{2}$ decreases from this value, the value of $\tan \psi$ when $\Delta=\pi$ is now less than the value for the bare surface, and approaches zero as $n_{2}$ approaches $n_{2 p}^{-}$. At this point $\Delta$ begins to oscillate between a maximum and minimum value, which are $\pi / 2$ for $n_{2}=n_{2 p}^{-}$. Finally, for $n_{2}$ between $n_{2 p}^{-}$and $n_{3}$ curves such as those for $n_{2}=1.36$ in figure 3 are obtained, becoming smaller and smaller as $n_{2}$ approaches $n_{3}$.

It will be clear that the $\rho$ curves completely cover the plane, those with $n_{2}>n_{2 s}$ covering the ball plane to the right of the bare-surface point and those with $n_{2}<n_{2 s}$ covering the hall plane to the left of the baresurface point. Thus every conceivable state of polarization of the reflected light is possible when the incident light is linearly polarized at an azimuth of $45 \mathrm{deg}$. Linearly polarized light at all possible azimuths is represented by the intercepts on the horizontal axis, and this occurs for the quarter-wave thickness. Circularly polarized light is obtained for that refractive index and thickness for which $\tan \psi=1$ and $\Delta= \pm \pi / 2$, and so forth. The efficacy of any of these conditions must, however, be determined by calculations such as those carried out for the special cases $n_{2 s}$ and $n_{2 p}^{-}$.

The curves of figure 2 and 3, while illustrating the dependence of $\rho$ upon thickness, do not show clearly the dependence of $\tan \psi$ and $\Delta$ on thickness. In figure 4 we show how tan $\psi$ varies with $d$ for several values of $n_{2}$. All these curves are periodic in $2 n_{2} d \cos \theta_{2} / \lambda$. However, since we have chosen certain critical values of $n_{2}$, the curves are not as symmetric as those previously described [2]. The values of $n_{2}$ chosen are as follows: two (1.41485 and 1.41600) close to $n_{2 s}$, one (1.3786) at $n_{2 p}^{-}$, one at the value of $n_{2}$ (1.3942) for which $\tan \psi$ at $\Delta=\pi$ is equal to $\tan \psi$ for the bare surface, and one value of $n_{2}$ (1.50) greater than $n_{2 s}$. Considering first the case where $n_{2}=n_{2 p}^{-}$, it will be seen that at $d=3500 \AA$, $\tan \psi=0$, and hence the reflected light is polarized with the electric vector in the plane of the surface. Similarly, for $n_{2}$ values slightly lower and slightly bigher than $n_{2 s}$ (1.41485 and 1.41600, respectively) tan $\psi$ becomes extremely large when the thickness is the quarterwave thickness. Since the sensitivity (which may be defined as the change of $\psi$ with thickness $[3,15])$ is the slope of these curves, it will be recognized that $n_{2}$ values near $n_{2 s}$ and thickness values around the quarter-wave thickness give the highest sensitivity. Moreover, the curves for $n_{2}=1.3786$ and 1.41485 and 1.41600 again show the difficulty of constructing a polarizer using films of these critical refractive indexes; the change of $\tan \psi$ with thickness near the proper thickness is almost discontinuous, so that to make a worthwhile polarizer using these effects would be very difficult indeed.

In figure 4 we have also shown the curve for the value of $n_{2}$ for which $\tan \psi$ has the least variation with thickness, with the exception of values of $n_{2}$ very close to $n_{1}$ or $n_{3}$. This is the value of 1.3942 , and for this value, tan $\psi$ when $\Delta=\pi$ (the quarterwave thickness) is equal to the value of $\tan \psi$ for the bare surface. As can be seen from figure 3 this $\rho$ curve is almost circular and centered at the origin. The total variation in tan $\psi$ is from 0.743 to 0.650 , or, in degrees, from 36.66 to 33.0 ; this change of $3.7 \mathrm{deg}$ is to be compared with the change of 45 deg obtained for $n_{2}=n_{2 s}$ or $n_{2}=n_{2 p}^{-}$.

In figures 5 and 6 we illustrate the behavior of $\Delta$ with thickness for the same values of $n_{2}$ shown in figure 4. For $n_{2}>n_{2 s}$ or $n_{2}<n_{2 p}^{-}, \Delta$ oscillates between two values. This behavior is typified by the curve for $n_{2}=1.50$ (which is, however, somewhat more asymmetric than previously shown curves) and has been illustrated many times [2]. As $n_{2}$ approaches $n_{2 s}$ from above or $n_{2 p}^{-}$from below, the curve of $\Delta$ versus thickness becomes more and more asymmetric, finally approaching a discontinuous curve where $n_{2}=n_{2 p}^{-}$or $n_{2 s}$. This is illustrated by the curves for $n_{2}=1.3786$ and 1.41600 in figure 5 . In these curves as the "half-wave" thickness is passed, $\Delta$ suffers a very sharp change from $-\pi / 2$ to $\pi / 2$, and since $\psi$ under these conditions is close to either zero or $\pi / 2$, the ellipse which represents the polarization of the reflected light goes very rapidly from a very thin ellipse with one sense of rotation to a similar ellipse with the opposite sense of rotation.

The behavior is decidedly different for $n_{2 p}^{-}<n_{2}<n_{2 s}$. Under these conditions $\Delta$ takes on all values from $2 \pi$ to 0 . This is illustrated in figure 6 for the refractive indices 1.41485 and 1.3942 . The value of 1.41485 is slightly less than $n_{2 s}$, and the curve of $\Delta$ versus thickness for this value is to be compared with than for $n_{2}=1.41600$ in figure 5 . In the present case, $\Delta$ starts from $2 \pi$, approaches $3 \pi / 2$ as the thickness approaches the quarter-wave value, but then drops almost discontinuously to $\pi / 2$ as the thickness becomes greater than the quarter-wave value. The curve for $n_{2}=1.3942$ illustrates the behavior for intermediate values of $n_{2}$ between $n_{2 p}^{-}$and $n_{2 s}$. As $n_{2}$ approaches $n_{2 p}^{-}$, again the curve of $\Delta$ versus thickness becomes more and more asymmetric, approaching a discontinuous curve as $n_{2}$ approaches $n_{2 p}^{-}$.

We have finally to discuss the intensity of the reflected light for these various situations. This is shown in figure 7 as a function of thickness for the same refractive indexes used for figures 2 to 6 . The reflectivity shown here is calculated for "ellipsometric" conditions, i.e., the incident light is linearly polarized at an azimuth of $45 \mathrm{deg}$ to the plane of incidence, and is given by $\left(r_{p}{ }^{2}+r_{s}{ }^{2}\right) / 2$. Starting from 


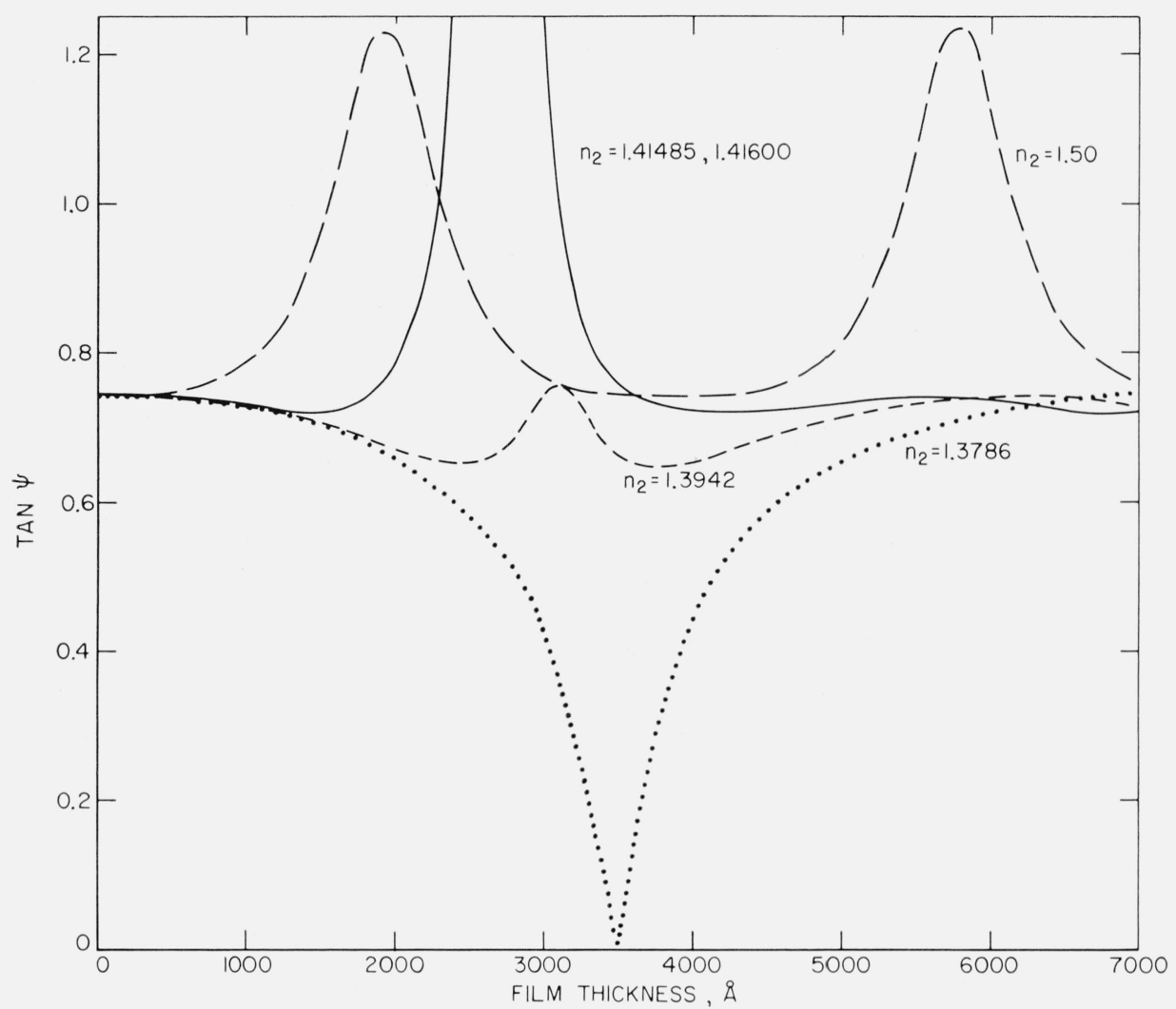

Figure 4. Case 1. Dependence of tan $\psi$ on film thickness for several values of $\mathrm{n}_{2}$ shown in figures 2 and 3.

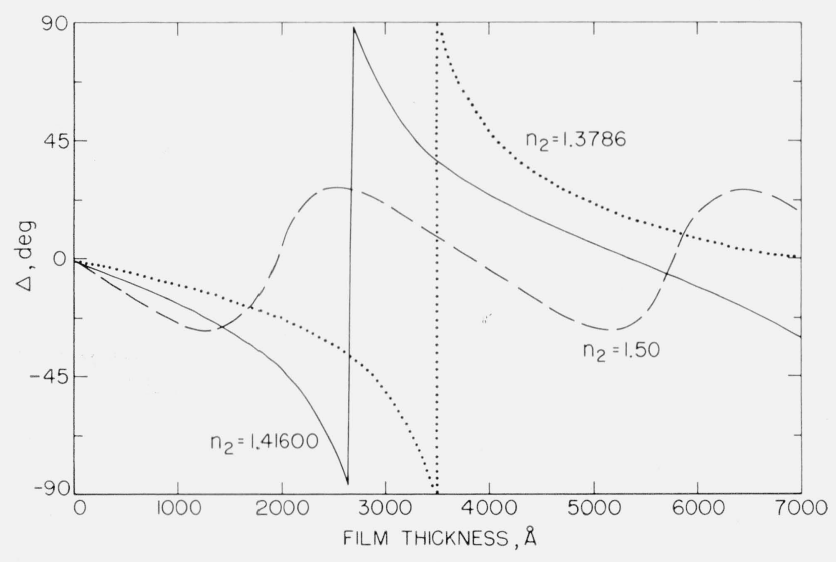

Figure 5. Case 1. Dependence of $\triangle$ on film thickness for the three values of $\mathrm{n}_{2}$ shown in figure 4 for which $\mathrm{n}_{2} \geq \mathrm{n}_{2 \mathrm{~s}}$ or $\mathrm{n}_{2} \leq \mathrm{n}_{2 \mathrm{p}}^{-}$.

a bare-surface value of 41 percent, the reflectivity drops to a minimum value at the quarter-wave thickness. For $n_{2}$ close to $n_{2 s}$ or $n_{2 p}$ this minimum value is very low indeed, being of the order of 2 to 3 percent. With the present values of refractive index for $n_{1}$ and $n_{3}$, production of polarized light by such dielectric films would be an inefficient process. More propitious values of $n_{1}$ and $n_{3}$ do, however, give higher reflectivity, at least for $n_{2 p}^{+}[12]$. In-

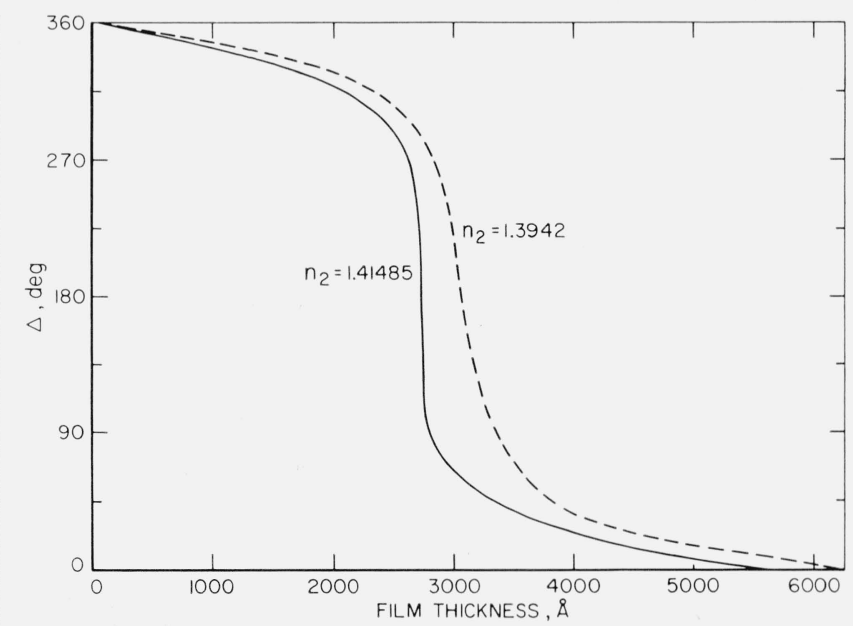

Figure 6. Case 1. Dependence of $\triangle$ on film thickness for the two values of $\mathrm{n}_{2}$ shown in figure 4 for which $\mathrm{n}_{2 \mathrm{p}}^{-}<\mathrm{n}_{2}<\mathrm{n}_{2 \mathrm{~s}}$.

creasing the angle of incidence to $46.60 \mathrm{deg}$ (only $0.08 \mathrm{deg}$ less than $\theta_{c}^{1,3}$ ) gave $n_{2 s}=1.3679$, increased the bare-surface reflectivity to 72.8 percent, but increased the minimum reflectivity to only 3.3 percent. Nevertheless, for films less than about $1000 \AA$ the reflectivity for an angle of incidence of $46.00 \mathrm{deg}$ is 30 percent or greater, and hence the intensity of reflected ligh is sufficient for experimental measurements under chese conditions. 


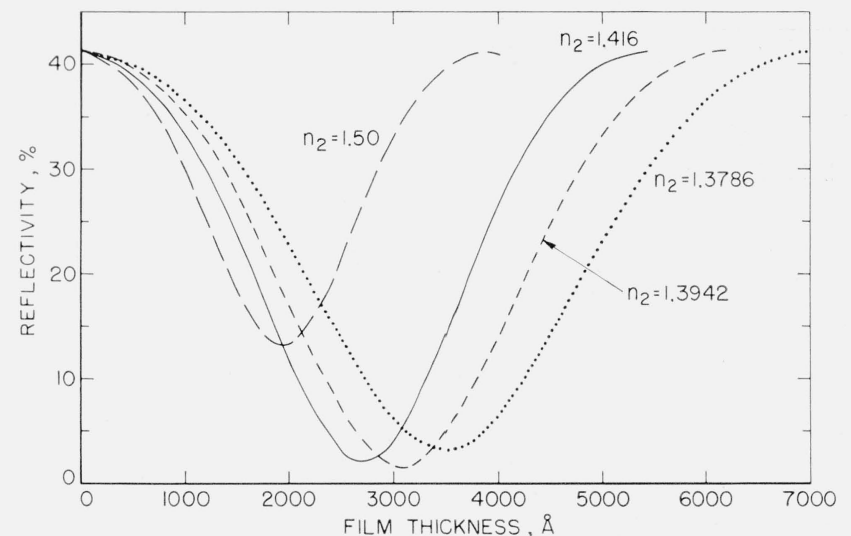

Figure 7. Case 1. Dependence of reflectivity on film thickness for several values of $\mathrm{n}_{2}$ shown in figures 2 to 6 .

The reflectivities are calculated for incident light at 45 deg azimuth with respect to the plane of incidence.

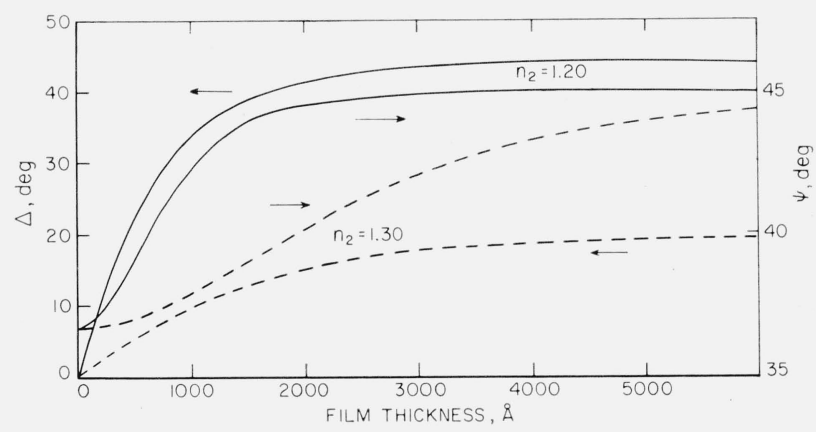

Figure 8. Case 2. Frustrated total reflection. Variation of $\Delta$ and $\psi$ with film thickness.

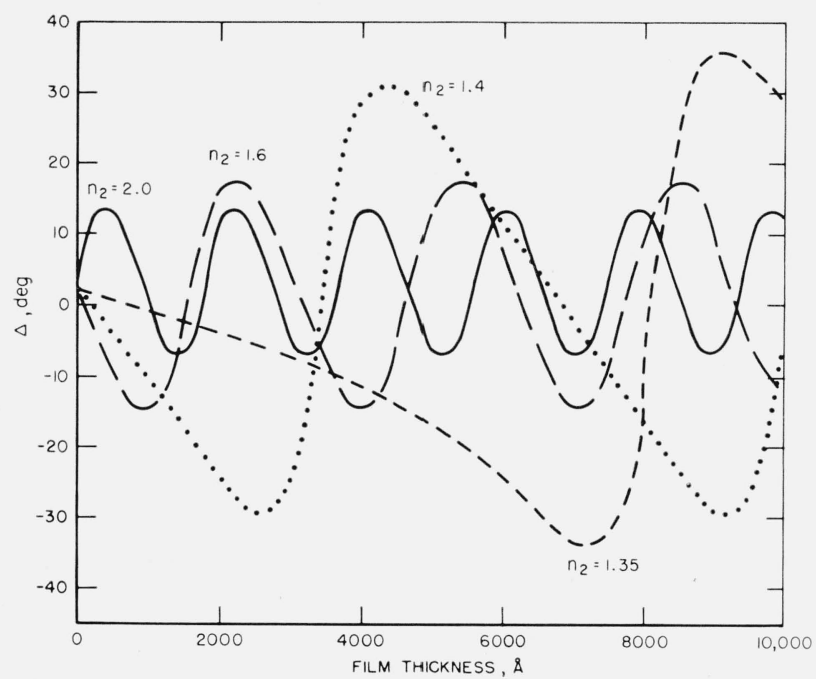

Figure 9. Case 3. Dependence of $\triangle$ on film thickness for several values of $\mathrm{n}_{2}$, including the condition $\mathrm{n}_{2}>\mathrm{n}_{1}$.

5. Case 2, $\theta^{1,3}>\theta_{i}>\theta_{c}^{1,2}$

This case is known as "Frustrated Total Reflection" and has already been extensively treated [16].
For this case $n_{2}$ must be less than $n_{3}$, and $\Delta$ and $\psi$ are shown in figure 8 as a function of film thickness for the two values of $n_{2}$ given in table 1 . For large film thicknesses $\tan \psi$ must approach unity, and $\Delta$ approaches some value determined by the angle of incidence and the refractive indexes in question. For $n_{2}=1.20$ this value of $\Delta$ is $44 \mathrm{deg}$, while for $n_{2}=$ $1.30 \mathrm{it}$ is $19.5 \mathrm{deg}$ for this angle of incidence $(46.0 \mathrm{deg})$. For very thin films whose refractive index is such as to make the present conditions apply, it is possible to measure thickness and refractive index by a single measurement of $\Delta$ and $\psi$. For the present conditions the minimum reflectivity (occurring when the films are of zero thickness) is 41 percent, and this rises as the film increases in thickness.

\section{Case 3, $\theta_{c}^{1,3}<\theta_{i}<\theta_{c}^{1,2}$}

For this case $n_{2}>n_{3}$, and $\tan \psi$ is always unity. The reflectivity is therefore always 100 percent. However, the only experimental parameter which varies is $\Delta$, and this permits only the evaluation of $n_{2}$ or $d$, but not both; one must be known from other considerations. The behavior of $\Delta$ with film thickness is shown in figure 9 for several values of $n_{2}$. As in case $1, \Delta$ oscillates between maximum and minimum values with a periodicity of $\left(2 n_{2} d \cos \theta\right) / \lambda$. However, since $\tan \psi$ is always unity none of the interesting polarization effects that occurred in case 1 occur here. As is evident from figure $9, \Delta$ is more sensitive to thickness for those refractive indexes which make $\theta_{c}^{1,2}$ close to the angle of incidence. The curves of $\Delta$ against thickness for these refractive indexes (1.40 and 1.35 in fig. 9) are asymmetric as were the curves in figure 5. We have calculated for this case the behavior of $\Delta$ with film thickness for one value of $n_{2}>n_{1}\left(n_{2}=2.0\right)$, and except for the fact that the "phase" of this curve is different from those with $n_{2}<n_{1}$, the behavior is very similar.

\section{Case $4, \theta_{c}^{1,3}<\theta_{i}>\theta_{c}^{1,2}$}

This is the case which is easiest to achieve in practice [10], for all that it necessitates is that $\theta_{i}$ be sufficiently high. As in case 3 only $\Delta$ varies with film thickness and thus from a single determination of $\psi$ and $\Delta$ only the refractive index or thickness of the film may be determined; one must be known from other considerations. Figure 10 shows the result of computations of $\Delta$ as a function of film thickness for the three cases shown in table 1 . As expected, the curves level off and show none of the periodicity present in cases 1 and 3 . It is to be noticed that $\Delta$ rises with film thickness for $n_{2}<n_{3}$ and falls for $n_{2}>n_{3}$. Thus it is not strictly correct to say that only thickness or refractive index can be determined; by comparing the value of $\Delta$ for the film-covered surface with that for the bare surface one can determine whether the film has a refractive index which is greater or less than the refractive index of the medium 3. Beyond this one cannot in general say from a single measurement. 


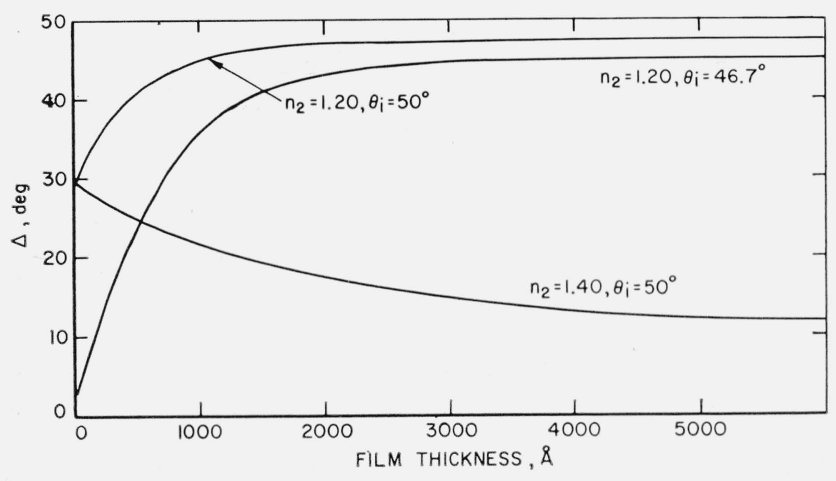

Figure 10. Case 4. Dependence of $\triangle$ on film thickness for $\mathrm{n}_{2}<\mathrm{n}_{3}$ and $\mathrm{n}_{2}>\mathrm{n}_{3}$.

\section{Sensitivity}

We have spoken of the desirability in cases 1 and 2 of using an angle of incidence near $\theta_{c}^{1,3}$ in order to increase reflectivity. There are other criteria, however, which influence the choice of angle of incidence. In case 3 and case 4 , in particular, where total reflection occurs, there is the question of experimental sensitivity. This may be defined as the change in $\Delta$ for a given change in film thickness $[3,15]$, and, for very thin films, a determination of this quantity as a function of angle of incidence leads to an unequivocal result. In figure 11 we show the change in $\Delta$ for a $100 \AA$ film as a function of angle of incidence for $n_{2}$ values of $1.20,1.40$, and 1.60. Two of the curves span cases 1, 3, and 4, and one spans cases 2 and 4. For each of the curves it is seen that the sensitivity rises as the angle of incidence is lowered. Without considerations of intensity one would conclude that the lowest angle of incidence should be used. However, with an angle of incidence less than $\theta_{c}^{1,3}$ the conditions are those of case 1 or 2 , and reflectivity falls off very rapidly as the angle of incidence is lowered. (The minimum angle of $44^{\circ}$ in this graph was chosen to give a reflectivity of about 17 percent for zero film thickness.) Balancing reflectivity against sensitivity one is led to the conclusion that it is always best to work as close to $\theta_{c}^{1,3}$ as possible. If $\theta_{i}$ is slightly less than $\theta_{c}^{1,3}$ then, for thin films such as these, one has the added advantage of having $\psi$ also vary with film thickness. When $n_{2}>n_{3}$, choosing $\theta_{i}$ slightly above $\theta_{c}^{1,3}$ gives optimum sensitivity and complete reflectivity, although only $\Delta$ varies. While it is not our purpose to pursue the effect of $n_{2}$ on sensitivity, it will be clear that sensitivity will be zero for $n_{2}$ equal to $n_{1}$ or $n_{3}$, be a maximum for some value of $n_{2}$ between $n_{1}$ and $n_{3}$, and again increase for $n_{2}>n_{1}$ or $n_{2}<n_{3}$. This is the reason that the curves for $n_{2}$ of 1.20 and 1.60 in figure 11 are higher than the curve for an $n_{2}$ of 1.40 .

\section{Appendix}

It will be shown that for reflection from a film covered surface either $r^{p}$ or $r^{s}$ may be zero, and hence $\tan \psi$ may be either zero or infinity.

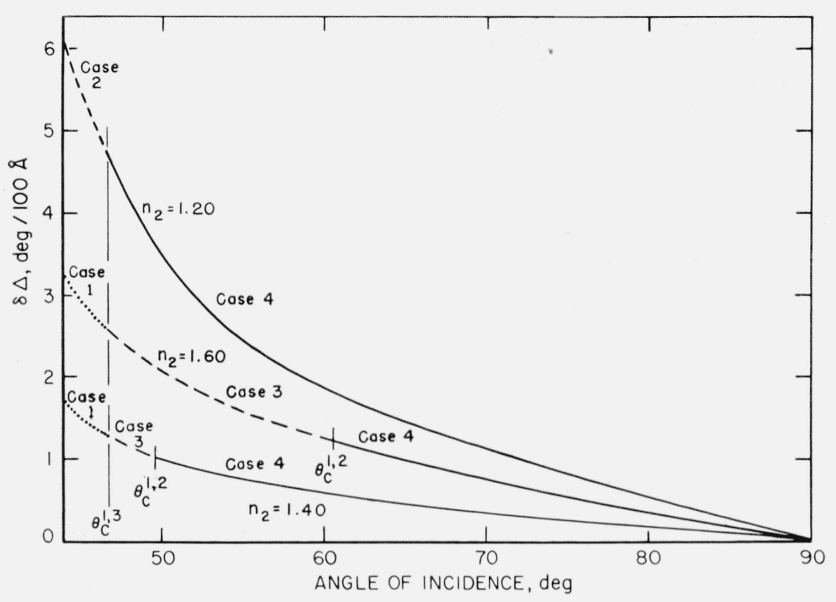

Figure 11. Sensitivity in $\triangle$ as a function of $\theta_{i}$ for three values of $\mathrm{n}_{2}$.

Each of the curves spans at least two cases. Solid line: case $4-$ case 3 ; - - case $2 ;.$. . case 1 .

The reflection coefficient $r^{\nu}$, where $\nu$ represents either $s$ or $p$ is given by

$$
r^{\nu}=\frac{r_{12}^{\nu}+r_{23}^{\nu} \exp D}{1+r_{12}^{\nu} r_{23}^{\nu} \exp D}
$$

where the terms have the same meaning as in eq (2). In order for this reflection coefficient to vanish, two conditions must be met:

$$
\begin{aligned}
& D=-i \pi ; \frac{2 n_{2} d \cos \theta_{2}}{\lambda}=\frac{k}{2} \\
& r_{12}^{\nu}=r_{23}^{\nu}
\end{aligned}
$$

where $k$ is an odd integer. For these conditions satisfied and $\nu=p, \tan \psi=0$; for $\nu=s, \tan \psi$ is infinite. The first state has been studied already [11, 12] but we shall be interested in more detail. We shall discuss each of the two states in turn.

State 1-vanishing of component with electric vector in plane of incidence

For this state, $\nu=p$, and the Fresnel coefficients are given by

$$
r_{i j}^{p}=\frac{n_{j} \cos \theta_{i}-n_{i} \cos \theta_{j}}{n_{j} \cos \theta_{i}+n_{i} \cos \theta_{j}}
$$

where $\theta_{j}$ is the angle between the normal to the $i, j$ interface and the ray in medium $j$. Substituting this expression into eq (A3) and simplifying leads to the following equation for $n_{2 p}$, the critical value of $n_{2}$ necessary to make the $p$ component vanish:

$$
n_{2 p}^{2}=\frac{1}{2} \frac{n_{1} n_{3}}{\cos \theta_{1} \cos \theta_{3}}\left[1 \pm\left(1-\sin 2 \theta_{1} \sin 2 \theta_{3}\right)^{1 / 2}\right]
$$

This equation has already been derived by Schröder [12] in slightly different form. There are two solu- 
tions, which we call $n_{2 p}^{+}$and $n_{2 p}^{-}$, and we shall investigate certain of their properties.

The critical value $n_{2 p}$ is symmetrically dependent upon $n_{1}, n_{3}, \theta_{1}$, and $\theta_{3}$. At normal incidence, $\left(\theta_{1}=0, \theta_{3}=0\right)$, we obtain the well-known solution [11]

$$
n_{2 p}^{+}=\left(n_{1} n_{3}\right)^{1 / 2}
$$

for the positive solution, and $n_{2 p}^{-}=0$ for the negative solution. The latter is, of course, inadmissible. For $n_{1}<n_{3}, \theta_{1}$ may take values up to $\pi / 2$, and as $\theta_{1}$ approaches $\pi / 2, n_{2 p}^{+}$increases without bound. For $n_{1}>n_{3}, \theta_{3}$ approaches $\pi / 2$ as $\theta_{1}$ approaches $\theta_{c}^{1,3}$, the critical angle for total internal reflection, and $n_{2 p}^{+}$ increases without limit as $\theta_{1}$ approaches $\theta_{c}^{1,3}$. It may be shown [12] that the behavior of $n_{2 p}^{+}$as a function of $\theta_{1}$ is monotonic. Hence, for the positive solution

$$
n_{2 p}^{+} \geq\left(n_{1} n_{3}\right)^{1 / 2}
$$

the equality sign holding at normal incidence.

For the parameters studied here, i.e., $\theta_{1}=46^{\circ}$, $n_{1}=1.8376, n_{3}=1.337, n_{2 p}^{+}=4.654$. For this value of $n_{2 p}^{+}, d_{0}$ is calculated from eq $(\mathrm{A} 1)$ as $304 \AA$ for $\lambda=5461 \AA$.

For the negative solution, $n_{2 p}^{-}=0$ at normal incidence, and becomes $\left(n_{1} n_{3} \sin \theta_{3}\right)^{1 / 2}$ at grazing incidence. The value of $n_{2 p}^{-}$is always less than $\left(n_{1} n_{3}\right)^{1 / 2}$, which in many cases of interest is too low to be realized experimentally. For the present case, at an angle $\theta_{1}$ of $46^{\circ}, n_{1}=1.8376$ and $n_{3}=1.337, n_{2 p}^{-}=$ 1.3786 , which is easily attainable. For this refractive index, the critical thickness is $3490 \AA$ for $\lambda=5461 \AA$, and $r^{s}=0.25$.

It is of interest to calculate the value of $\theta_{i}$ for which $n_{2 p}^{-}$is unity. This, of course, represents the minimum angle of incidence for a given $n_{1}$ and $n_{3}$ for which the value of $n_{2 p}^{-}$is admissible. A certain amount of algebraic manipulation gives

$$
\begin{aligned}
\sin ^{2} \theta_{1}= & \frac{n_{3}^{2}+n_{1}^{2}-2 n_{1}^{4} n_{3}^{4}}{2 n_{1}^{2}\left[1-n_{1}^{4} n_{3}^{4}\right]} \\
& -\frac{\left[\left(n_{3}^{2}-n_{1}^{2}\right)^{2}+4 n_{1}^{4} n_{3}^{4}\left(n_{1}^{2}-1\right)\left(n_{3}^{2}-1\right)\right]^{1 / 2}}{2 n_{1}^{2}\left[1-n_{1}^{4} n_{3}^{4}\right]} .
\end{aligned}
$$

Except for the presence of the term $n_{1}^{2}$ in the denominator this expression is symmetrical in $n_{1}$ and $n_{3}$. Angles of incidence less than the value given by this equation cannot produce linearly polarized light with the electric vector normal to the plane of incidence for the negative solution to eq (A5).

State 2-vanishing of component with electric vector normal to plane of incidence

Although mentioned by Schröder [12], this case does not appear to have been studied. Indeed, it has been considered impossible [11]. For this case the Fresnel coefficient is

$$
r_{i j}^{s}=\frac{n_{i} \cos \theta_{i}-n_{j} \cos \theta_{j}}{n_{i} \cos \theta_{i}+n_{j} \cos \theta_{j}} .
$$

Substituting this in eq (A3) leads to the following equation for the critical value of $n_{2}$ for the reflected light to be polarized entirely with its electric vector in the plane of incidence $\left(n_{2 s}\right)$ :

$$
n_{2 s}^{2}=n_{1} n_{3} \cos \theta_{1} \cos \theta_{3}+n_{1}^{2} \sin ^{2} \theta_{1} .
$$

In this case there is only one value of $n_{2 s}$ which is admissible. At normal incidence we again obtain $n_{2 s}=\left(n_{1} n_{3}\right)^{1 / 2}$.

Not all combinations of $n_{1}, n_{2}$, and $n_{3}$ are possible in this case. To show this we recast eq (A9) into the following form

$$
\frac{n_{2}^{2}}{n_{1}^{2}}=\sin ^{2} \theta_{1}\left[\frac{1}{\tan \theta_{1} \tan \theta_{3}}+1\right]
$$

Now, if $n_{2}>n_{1}$, then

$$
\sin ^{2} \theta_{1}\left[\frac{1}{\tan \theta_{1} \tan \theta_{3}}+1\right]>1
$$

or

$$
\tan \theta_{3}<\tan \theta_{1}
$$

and hence $n_{3}>n_{1}$.

A similar calculation for $n_{2}<n_{1}$ leads to the condition $n_{3}<n_{1}$. An obvious way to satisfy these conditions is for $n_{1}, n_{2}$, and $n_{3}$ either to increase or decrease in order. For the case of $n_{1}=1.337, n_{3}=1.8376$, $\theta_{1}=46^{\circ}$ (which is the opposite of the direction of propagation treated in the text), direct computation gives $n_{2}=1.543$ and $d_{2}=1130$ for $\lambda=5461$. For these values the reflected light is polarized entirely with its electric vector in the plane of incidence. The value of $r^{p}$ is, however, only 0.032. For the opposite direction of propagation $\left(n_{1}=1.8376, \quad n_{3}=1.337\right)$ $n_{2}=1.41542$.

\section{References}

[1] P. Drude, Ann. Physik. 272, 532 (1889); ibid 272, 865 (1889); ibid 275, 481 (1890).

[2] A. B. Winterbottom, Optical studies of metal surfaces, The Royal Norwegian Scientific Society Report No. 1, 1955 (F. Bruns, Trondheim, Norway).

[3] F. L. McCrackin, E. Passaglia, R. R. Stromberg, and H. L. Steinberg, J. Res. NBS 6rA (Phys. and Chem.), No. 4, 363 (1963)

[4] For a State of the Art, see papers in Ellipsometry in the measurement of surfaces and thin films (Symposium Proceedings), edited by E. Passaglia, R. R. Stromberg, and J. Kruger, NBS Misc. Publ. No. 256 (1964).

[5] F. L. McCrackin and J. P. Colson, ibid, p. 61.

[6] N. J. Harrick, Ann. N. Y. Acad. Sci. 101 Art. 3, 928 (Jan. 1963); J. Phys. Chem. 64, 1110 (1960).

[7] J. Fahrenfort, Spectrochim. Acta. 17, 698 (1961).

[8] R. R. Stromberg, E. Passaglia, and D. J. Tutas, J. Res. NBS 67A (Phys. and Chem.), No. 5, 431 (1963).

[9] F. L. MeCrackin and J. Colson, NBS Tech. Note 242 (1964).

[10] D. J. Tutas, R. R. Stromberg, and E. Passaglia, Soc. Plastics Eng. Trans. 4, 256 (1964).

[11] M. Born and E. Wolf, Principles of Optics, p. 60 (Pergamon Press, New York, N. Y., 1959).

[12] H. Schröder, Optik. 3, 499 (1948).

[13] R. E. Hartman, J. Opt. Soc. Am. 44, 192 (1954).

[14] R. D. Mattuck, J. Opt. Soc. Am. 46, 615 (1956).

[15] R. C. Smith and M. Hacksaylo, see reference 4, p. 83.

[16] T. R. Young and J. M. Fath, see reference 4, p. 349.

(Paper 68A6-307) 